\title{
MATHEMATICAL MODELING OF FILLING GAS CENTRIFUGE CASCADE FOR NICKEL ISOTOPE SEPARATION BY VARIOUS FEED FLOW RATE Ushakov A.A. ${ }^{1,2}$, Orlov A.A. ${ }^{2}$, Sovah V.P. ${ }^{1}$ \\ ${ }^{1}$ Joint Stock Company «Production Association «Electrochemical Plant» \\ ${ }^{2}$ National Research Tomsk Polytechnic University E-mail: ushakovaa2015@sibmail.com
}

During the operation of gas centrifuge (GC) cascade for the multicomponent isotope mixture (MIM) separation there are nonstationary hydraulic processes. In all nonstationary processes, safe operation of equipment should be ensured and separation work losses should be minimized. In this regard, the research into the effects of nonstationary processes on the efficiency of MIM separation seems to become even more important. We had done verification of developed mathematical model as an example silicon, krypton and germanium isotope separation. Filling GC cascade with process gas precedes an isotope separation mode. Distribution of isotopes in cascade stages after its filling is initial conditions for calculation of subsequent nonstationary process. Thus actual task is research MIM separation during filling GC cascade. Earlier we had identified that isotope concentrations on light and heavy fraction flows after filling cascade depend on feed stage number.

This article presents the results of research filling GC cascade for nickel isotope separation with process gas by various feed flow rate. Nickel isotopes are used for nuclear physics experiments and production radioactive isotopes (for example, $62 \mathrm{Ni}$ stable isotope is used as source material to produce $63 \mathrm{Ni}$ radioactive isotope). Analysis of the research results has shown that nickel isotopes are distributed along the cascade length in accordance with their mass numbers regardless of feed flow rate. Isotope concentrations in stages, light and heavy fraction flows after filling cascade depend on feed flow rate. The increase of feed flow rate leads to reduce of isotope concentration maximum value and the transfer of isotopes. Optimization of feed flow rate during filling cascade makes it possible to reduce duration of the subsequent nonstationary process to steady-state mode of cascade. 